\title{
Detection of knots and jets in IRAS 06061+2151
}

\author{
B. G. Anandarao ${ }^{1}$, A. Chakraborty ${ }^{2}$, D. K. Ojha ${ }^{3,5}$, and L. Testi ${ }^{4}$ \\ 1 Physical Research Laboratory, Ahmedabad-390009, India \\ 2 Department of Astronomy \& Astrophysics, 525 Davey Laboratory, Pennsylvania State University, University Park, \\ PA 16802, USA \\ 3 Tata Institute of Fundamental Research, Homi Bhabha Road, Mumbai-400005, India \\ 4 Osservatorio Astrofisico di Arcetri, INAF, Largo E. Fermi 5, 50125 Firenze, Italy \\ 5 National Astronomical Observatory of Japan, Mitaka, Tokyo 181-8588, Japan
}

Received 26 November 2003 / Accepted 15 March 2004

\begin{abstract}
We report detection of a Young Stellar Object with evidence of outflow in the form of knots in the molecular hydrogen emission line $(2.121 \mu \mathrm{m})$ towards the massive star forming region IRAS 06061+2151. Near-infrared images reveal IRAS $06061+2151$ to be a cluster of at least five sources, four of which seem to be early B type young stellar objects, in a region of 12 arcsec surrounded by a nebulosity. The presence of the knots that are probably similar to the HH objects in the optical wavelengths suggests emerging jets from one of the cluster members. These jets appear to excite a pair of knot-like objects (Knot-NW and Knot-SE) and extend over a projected size of $0.5 \mathrm{pc}$. The driving source of the jets is traced back to a member of the cluster whose position in the $H-K \mathrm{~s} / J-H$ color-color diagram indicates that it is a Class I type pre-main sequence star. We also obtained $K$ band spectra of the brightest source in the cluster and of the nearby nebular matter. The spectra show molecular hydrogen emission lines but do not show the $\operatorname{Br} \gamma$ line $(2.167 \mu \mathrm{m})$. These spectra suggest that the excitation of the molecular hydrogen lines is probably due to a mild shock.
\end{abstract}

Key words. stars: pre-main sequence - ISM: jets and outflows - stars: formation

\section{Introduction}

IRAS $06061+2151$ is one of the most luminous IRAS sources believed to be associated with the galactic massive star forming region Gemini OB1 which is at a distance of $2 \mathrm{kpc}$ (Carpenter et al. 1995a). The object figures in the radio survey of massive star forming regions done by Kurtz et al. (1994, hereafter KCW94). The CO survey of Shepherd \& Churchwell (1996) categorized this object as having a moderate velocity gas flow (full width $=20.8 \mathrm{~km} \mathrm{~s}^{-1}$ and $F W H M=4 \mathrm{~km} \mathrm{~s}^{-1}$ ). This Ultra Compact HII(UCHII) region was previously studied by Carpenter et al. (1995a) and very recently in a near-infrared (NIR) survey on UCHII regions by Hanson et al. (2002, hereafter HLR02). The extensive CS survey and NIR observations by Carpenter et al. (1995a,b) of 58 IRAS sources in the Gemini OB1 molecular cloud complex suggested that more luminous IRAS sources tend to be associated with more massive cores. Based on their study, these authors proposed a qualitative model for the formation of massive dense cores, primarily through external compression that sweeps shells of molecular gas.

The mass outflows in the form of jets from young stellar objects (YSOs) are the important signatures of star formation

Send offprint requests to: B. G. Anandarao,

e-mail: anand@prl.ernet.in in molecular clouds (Hartmann 1998, and references therein). The jets in turn cause Herbig-Haro (HH) objects seen in optical emission lines, notably [SII] and $\mathrm{H} \alpha$, by shocking/interacting with the ambient interstellar medium. Such manifestations are better revealed in the NIR due to the lesser extinction that it suffers compared to the visible region. In the recent past, several discoveries have been made of molecular hydrogen outflows, both in situations where there exist optical counterparts in the form of HH objects (e.g., Schwartz et al. 1988; Davis et al. 1994; Eisloffel et al. 2000) and where there are no optical counterparts (e.g., Herbst et al. 1997). However, outflows detected in the case of massive proto-stars are rare due to the fact that the outflows are short-lived compared to those in the low mass proto-stars, though it is believed that stars of all masses might undergo these phenomena.

Therefore, luminous IRAS sources such as IRAS $06061+2151$ are appropriate candidates for further study in the NIR. Our motivation is to look for new outflows associated with massive protostars and to determine their evolutionary stages (such as Class I, II and III), using color-color diagrams (Lada \& Adams 1992; Lada et al. 1993; Gomez et al. 1994).

In this paper, we report detection of infrared counterparts of $\mathrm{HH}$ objects presumably caused by jets from a class I type source towards IRAS $06061+2151$. We study the nature of the 
cluster of sources using JHK photometry, narrowband images in molecular hydrogen $(2.12 \mu \mathrm{m})$ and $\mathrm{Br} \gamma$ lines and medium resolution $K$ band spectroscopy. The data were obtained by new observations as well as from the 2MASS archive. In Sect. 2 we present the details of observations and data reduction procedures, Sect. 3 deals with results and discussion and we summarize our conclusions in Sect. 4.

\section{Observations and data reduction}

\subsection{Observations from Mt. Abu}

$J(1.253 \mu \mathrm{m} / 0.296 \mu \mathrm{m}), H(1.659 / 0.288), K^{\prime}(2.120 / 0.360)$ photometric observations of IRAS $06061+2151$ were done from the $1.2 \mathrm{~m}$ PRL Infrared Telescope, Mt. Abu, India, using a $256 \times 256 \mathrm{HgCdTe}$ array (NICMOS-3, Infrared Laboratories, Arizona, USA). The instrument is described in Anandarao (1998) and Nandakumar (1999). The plate scale is $0.5^{\prime \prime} /$ pixel (corresponding to a field of view of $2^{\prime}$ ). The night of observation (26 Jan. 2001) was photometric with a seeing of $0.9-1.0^{\prime \prime}$.

Individual object frames were of $30 \mathrm{~s}$ of integration in $J$ and $H$ bands and $3 \mathrm{~s}$ in $K^{\prime}$ band. The data reduction was done using IRAF software tasks. All the images were subjected to standard pipeline procedures like dark and sky subtraction and flat-fielding. The images were then co-added to obtain a final image in each band $\left(J, H\right.$ and $\left.K^{\prime}\right)$ averaged over a total integration time of $300 \mathrm{~s}$. In each band two such images were obtained. The images were then filtered using a median filter of $3 \times 3$ pixels to remove noise at sky-level, thus making them suitable for photometric analysis. We used the DAOPHOT (Stetson 1987) tasks and followed the photometric procedure described by Chakraborty et al. (2000). We estimate the overall photometric error to be $\pm 0.08 \mathrm{mag}$. The faintest stars recorded were found to be of 16.66, 15.30 and 14.10 mag in $J, H$ and $K^{\prime}$ bands respectively.

\section{2. $2 M A S S$ data}

We extracted sources from the 2MASS All-Sky Point Source Catalogue(PSC) that were within $2^{\prime}$ radius of the IRAS source. Thus this region excludes the cluster G188.80+01.07 (see Bica et al. 2003). The data were downloaded from the 2MASS Homepage. The 2MASS point source catalogue consists of $J(1.24 \mu \mathrm{m} / 0.33 \mu \mathrm{m}), H(1.66 / 0.39)$ and $K \mathrm{~s}(2.16 / 0.42)$ magnitudes of stars. The PSC detection limits for a $S / N=10$ are $J=15.8, H=15.1$ and $K \mathrm{~s}=14.3$.

The 2MASS data are used to produce the color-color diagram to study the nature of sources. We use the Mt. Abu photometry for the five bright cluster candidate YSOs towards IRAS 06061+2151(see Sect. 3.1). The Mt. Abu magnitudes match reasonably well the 2MASS data as shown in Table 1 (except for Stars 1 and 5), despite the differences in the passbands.

\subsection{Observations from TNG, La Palma}

We obtained spectra $(R=1000,1.4$ to $2.4 \mu \mathrm{m})$ of one of the bright sources (Star No. 1) in the cluster of IRAS 06061+2151 using the Near Infrared Camera Spectrometer (NICS) attached to the $3.58 \mathrm{~m}$ Telescopio Nazionale Galileo (TNG) at La Palma. NICS has a HgCdTe Hawaii $1024 \times 1024$ array and it was used in the LF configuration with a plate scale of $0.25^{\prime \prime}$ (for instrument details, see Baffa et al. 2001). The medium resolution spectra were obtained using the HK grism with a slit width of $0.5^{\prime \prime}$. The Position Angle (PA) of the slit was 270 degrees i.e., in the East-West direction. Two dithered exposures of 60 secs each were obtained on Star No. 1. A hot star (A0) spectrum was also taken for telluric calibration. Following a similar procedure, spectra were obtained in a region $5^{\prime \prime}$ west of Star No. 1, in order to sample the nebular regions.

Apart from the spectra, we also obtained images of the source in two narrowband filters centered around $2.12 \mu \mathrm{m}$ for the $\mathrm{H}_{2} v=1-0 \mathrm{~S}(1)$ line and $2.17 \mu \mathrm{m}$ for the $\mathrm{Br} \gamma$ line. A total of five $60 \mathrm{~s}$ dithered exposures (dithered by more than 3 arcmin) were obtained in both the narrowband filters. The seeing was $0.8-0.9$ arcsec during observations.

The TNG images were analysed in a similar process as those from Mt. Abu. The spectra were reduced using standard IRAF tasks like APALL, TELLURIC, and normalized with respect to the respective continua. The wavelength calibration was done using the sky $\mathrm{OH}$ lines using the list of lines available with IRAF. We present here the $K$ band spectra for which the signal to noise ratio is better than 5 . This spectral region contains some of the most important features that we are looking for, such as the $\mathrm{Br} \gamma$ and $\mathrm{H}_{2}$ vibrational lines.

\section{Results and discussion}

\subsection{Photometry}

Figure 1 shows the $K^{\prime}$ band image of the central region of the object taken at Mt. Abu. The five prominent stellar sources (S1, 2, 3, 4 and 5) are marked on the figure and their magnitudes are given in Table 1. The data for the S4 (all three bands) and S5 (only in $K^{\prime}$ band) are from the Mt. Abu observations. S4 is detected in $H$ and $K$ s bands but not in $J$ band; S5 is detected only in $K$ s band with 2MASS. The Mt. Abu $K^{\prime}$ magnitude of S5 is 12.9 while in $J$ and $H$ bands the source was below the detection limit. HLR02 obtained an $H$ band image of the central region of this object which does not show S5, thus supporting our result. It appears then that this source has a rather steep spectral energy distribution in the NIR and beyond, hence the importance of the NIR study at high angular resolution (at least seeing-limited) of the massive star-forming regions.

The IRAS flux densities for $06061+2151$ are 12.0, 144.8, 865.6 and $1130 \mathrm{Jy}$ in the $12,25,60$ and $100 \mu \mathrm{m}$ bands respectively. From these values, Carpenter et al. (1995b) estimated the total far-infrared (FIR) luminosity to be $4000 L_{\odot}$. Based on the total FIR luminosity, Ghosh et al. (2000) suggested that the core may contain a single B0-B0.5 type hot star, though they could not resolve the cluster due to the large field of view (1.5 arcmin). Our NIR image (see Fig. 1), however, reveals that IRAS $06061+2151$ consists of a cluster of at least five stars.

Figure 2 shows $J-H / H-K$ s color-color diagram of sources from the 2MASS data and Mt. Abu observations (S1-S4). The 2MASS-PSC data covers a diameter of 4 arcmin around the 
Table 1. A comparison of stellar magnitudes from 2MASS data and Mt. Abu images.

\begin{tabular}{c|c|c|c|c|c|c|c}
\hline \hline No. & Co-ordinates (J2000) & \multicolumn{3}{|c|}{ 2MASS } & \multicolumn{3}{|c}{ Mt. Abu } \\
\cline { 3 - 8 } & RA-Dec & $J$ & $H$ & $K s$ & $J$ & $H$ & $K$ \\
\hline 1 & $6 \mathrm{~h} 9 \mathrm{~m} 7.2 \mathrm{~s}+21^{\circ} 50^{\prime} 40^{\prime \prime}$ & 15.46 & 12.87 & 11.22 & 15.54 & 12.68 & 10.85 \\
2 & $6 \mathrm{~h} 9 \mathrm{~m} 7.1 \mathrm{~s}+21^{\circ} 50^{\prime} 34^{\prime \prime}$ & 13.67 & 12.14 & 11.20 & 13.65 & 12.24 & 11.11 \\
3 & $6 \mathrm{~h} 9 \mathrm{~m} 7.5 \mathrm{~s}+21^{\circ} 50^{\prime} 32^{\prime \prime}$ & 15.31 & 12.94 & 11.55 & 15.44 & 13.05 & 11.45 \\
4 & $6 \mathrm{~h} 9 \mathrm{~m} 6.9 \mathrm{~s}+21^{\circ} 50^{\prime} 43^{\prime \prime}$ & - & 14.34 & 12.16 & 16.66 & 14.48 & 12.29 \\
5 & $6 \mathrm{~h} 9 \mathrm{~m} 7.1 \mathrm{~s}+21^{\circ} 50^{\prime} 32^{\prime \prime}$ & - & - & 13.52 & - & - & 12.90 \\
\hline
\end{tabular}

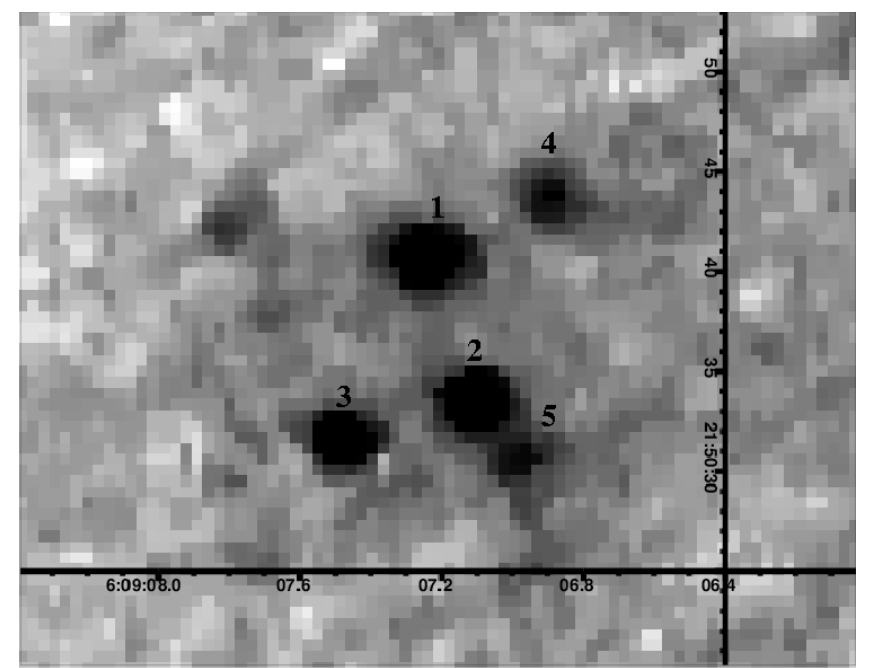

Fig. 1. $K^{\prime}$ band image of IRAS $06061+2151$ observed from Mt. Abu. The numbers represent the cluster of sources around IRAS $06061+2151$ region The abscissa and ordinate are in J2000.0 epoch.

co-ordinate of the IRAS source. The total number of sources extracted was 57, after removing all the "null" entries. The solid curve is the locus of points corresponding to unreddened main sequence stars and giants (Bessell \& Brett 1988). The two parallel lines (dashed) are the reddening vectors drawn up to the visual extinction of $A_{\mathrm{v}}=30$; together these parallel lines show the region in which the main-sequence stars occur at various extinctions (Hunter et al. 1995). Sources on the right side of the reddening strip $(\sim 17)$ are primarily stars with intrinsic color excess which include the S1-S4 stars of the cluster. We allowed an uncertainity of $0.1 \mathrm{mag}$ in $H-K$ color on either side of the strip to identify the left and right side of the reddening strip. Also shown in the figure is the locus for the CTTS (from Meyer et al. 1997) and the corresponding reddening vector.

The stars on the right side are mostly Young Stellar Objects (YSOs) (Lada \& Adams 1992; Lada et al. 1993; Gomez et al. 1994). In general, sources whose $H-K$ color is $\geq 1.5$ and $J-H \geq 1.0$ can be considered as Class I type YSOs or protostars in the early stages of their evolution (Strom et al. 1989; Kenyon et al. 1993; Lada \& Adams 1992). From Fig. 2, we find that out of the total of 17 stars falling clearly in the region on the right side of the reddening vector, 3-4 are clearly beyond the CTTS locus to its right. These stars that include $\mathrm{S} 4$ and maybe S2 can be classified as possible Class I candidates, if we account for the uncertainty of $0.1 \mathrm{mag}$ in colors. The rest, about 13-14 stars, can be classified as possible Class II-III. Thus, the region around IRAS 06061+2151 shows

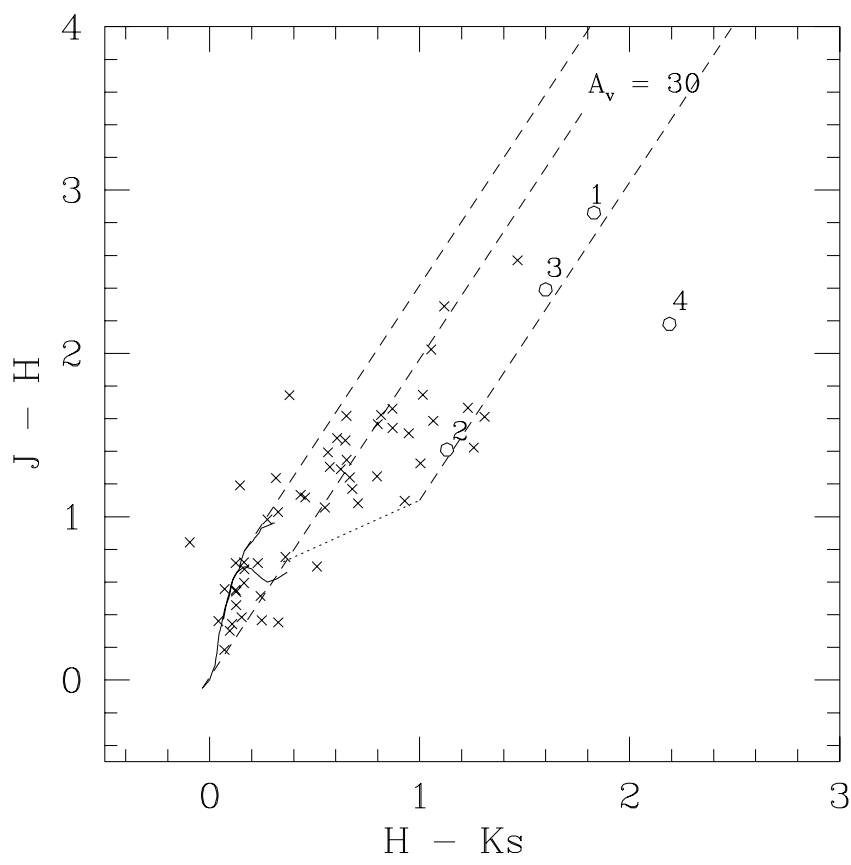

Fig. 2. Color-color diagram of the sources extracted from the 2MASSPSC data. The numbers represent the cluster of sources around IRAS $06061+2151$ region (see text and Table 1 for details). The solid line represents unreddened main-sequence stars and the giants (Bessell \& Brett 1988). The dashed lines are parallel to the reddening vector with magnitude of $A_{\mathrm{v}}=30$. The dotted line is the locus of CTTS (Meyer et al. 1997).

a concentration of YSOs. Indeed, NIR studies by Carpenter et al. (1995b) predicted a similar trend towards IRAS $06061+2151$.

Figure 3 shows the $\mathrm{H}-\mathrm{Ks} / \mathrm{Ks}$ color-magnitude diagram. As in Figs. 1 and 2 the stellar sources (S1, 2, 3 and 4) are marked. The solid line represents unreddened main-sequence stars (Koornneef 1983; Bessell \& Brett 1988) and the dashed line is the reddening vector of $A_{\mathrm{v}}=30 \mathrm{mag}$. S1 shows a high extinction of $A_{\mathrm{v}}=28$. The other sources, S2, S3 and S4, also show high extinctions of $A_{\mathrm{v}}=19,26$ and 36 respectively. Based on the photometry in Table 1, using the Rieke \& Lebofski (1985) extinction law and a distance modulus $=11.5 \mathrm{mag}$, the expected absolute $K$ magnitudes of the sources S1, 2, 3 and 4 are $M k=-3.6,-2.4,-2.8$ and -3.0 respectively. Source $\mathrm{S} 5$ which is only observed in the $K^{\prime}$ band perhaps suffers extinction greater than $A_{\mathrm{v}}=40 \mathrm{mag}$, if we consider that it is fainter than the detection limits of the Mt. Abu observations in $J$ and $H$ bands. 


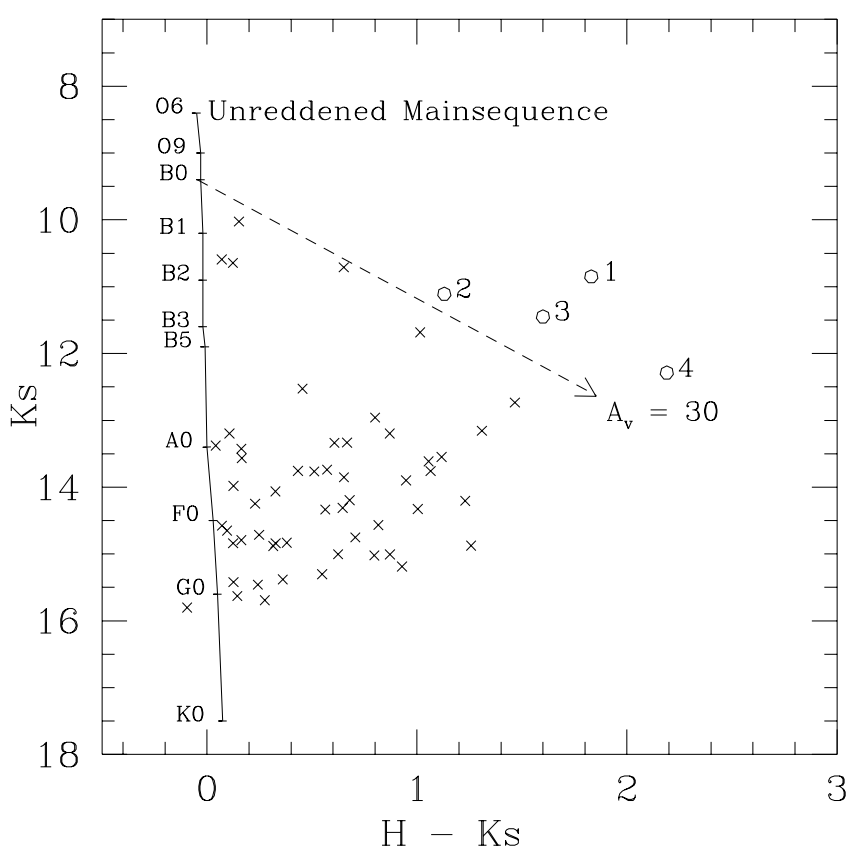

Fig. 3. Color-Magnitude diagram of the sources extracted from the 2MASS-PSC data. The numbers represent the cluster of sources around IRAS 06061+2151 region. The solid line represents unreddened main-sequence stars (Koornneef 1983; Bessell \& Brett 1988) and the dashed line is the reddening vector with magnitude of $A_{\mathrm{v}}=30$.

If the above arguments are true, then the sources have to be much more massive than T Tauri stars. Following the Palla \& Stahler (1999) evolutionary tracks, stars less massive than $3.5 M_{\odot}$ (at any age) are never more luminous than $M k=$ -1.67 . Assuming that more massive stars actually burn hydrogen while accreting, they are expected to have no pre-main sequence evolution (as the authors above advocate). Using the zero-age main sequence magnitude/color relations $M k=-3.6$ implies a spectral type between B0 and B1 and $M k=-2.4$ corresponds to B2. We have considered IR colors from Bessell \& Brett (1988) for our calculations. In all these arguments, we have neglected infrared excess and accretion luminosity.

For the source S1, being the most massive, the accretion luminosity could contribute substantially to the luminosity only if the accretion rate is very high. This is possible, but implies an amount of circumstellar material that would be so high as to produce a column density much higher than $A_{\mathrm{v}}=28 \mathrm{mag}$. For the other sources S2, 3 and 4 the uncertainities in the infrared excess will apply; however, the absolute magnitude-luminosity relation does give us a lower limit for the stellar masses.

The important finding of the current photometric study is Star S5 whose $J$ and $H$ mag are fainter than 15.2 and 16.4 mag (our detection limits) while the $K^{\prime}$ magnitude is 12.9 (2MASS data gives 13.52). The absence of S5 in the $H$ band image of HLR02 confirms this. A deeper NIR survey preferably at high spatial resolution could show exactly how steep the NIR energy distribution is for S5 and hence its exact nature. The difference in the $K$ magnitudes from Mt. Abu and 2MASS is 0.62 and is larger than the observational uncertainties. It is possible that the star is undergoing a variability that is akin to FU Orionis. Such

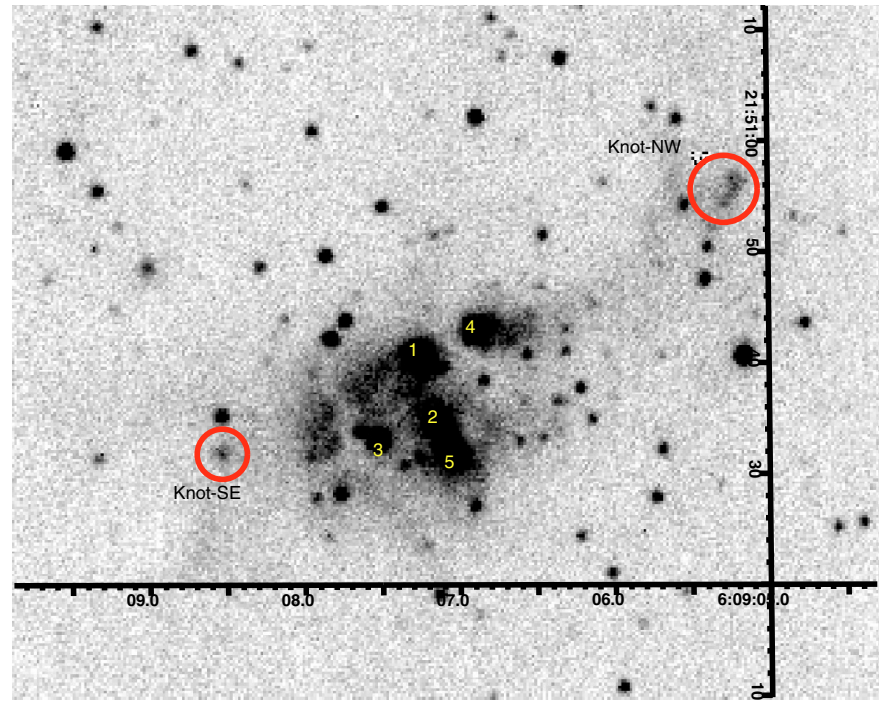

Fig. 4. $\mathrm{H}_{2}$ line + continuum $(2.122 \mu \mathrm{m})$ image observed from TNG. The circles show the Knot-like objects Knot-NW and Knot-SE. The numbers 1, 2, 3, 4 and 5 mark the positions of the stars as numbered in Table 1. North is up and East is to the left. The abscissa and ordinate are in $\mathrm{J} 2000.0$ epoch.

a substantial difference is also noticed (Table 1) in the case of the $K$ band for $\mathrm{S} 1$.

\subsection{Molecular hydrogen knots in IRAS $06061+2151$}

Figures 4 and 5 show narrow band images of the region in $\mathrm{H}_{2}$ $(2.121 \mu \mathrm{m}$, line + continuum $)$ and $\operatorname{Br} \gamma(2.167 \mu \mathrm{m}$, line + continuum). The circle marks two knot-like diffuse structures, one to the north-west and another to the south-east of the centre of the cluster in the $\mathrm{H}_{2}$ image. These two prominent diffuse emission features are not seen in the $\mathrm{Br} \gamma$ image. We may safely conclude then that the narrow band image at $2.167 \mu \mathrm{m}$ (Fig. 5) represents the background continuum radiation. We estimated the integrated brightness of the knots using relative photometry with respect to the $K$ s magnitude of S1 and S2. A large aperture of 20 arcsec was used for the knots. We find that in the $\mathrm{H}_{2} \mathrm{im}-$ age, the western knot is $1.6 \mathrm{mag}$ and the eastern knot $2.5 \mathrm{mag}$ fainter than S1. A similar estimation of brightness of faint stars seen in both the $\mathrm{H}_{2}$ and $\mathrm{Br} \gamma$ images showed that they are 4 mag fainter. We also find that these knot-like structures should be at least 4.5 mag fainter than $\mathrm{S} 1$ in the $\mathrm{Br} \gamma$ filter since they are not detected in the image.

We thus establish that the two newly identified structures are primarily due to $\mathrm{H}_{2}$ emission. The $\mathrm{H}_{2} v=1-0 \mathrm{~S}(1)$ $2.122 \mu \mathrm{m}$ line emission has proven to be an excellent tracer in dense molecular cloud regions for moderate shocks in the range of 10 to $30 \mathrm{~km} \mathrm{~s}^{-1}$ (Shull \& Beckwith 1982; Draine et al. 1983; Sternberg \& Dalgarno 1989) and is found to trace the termination of $\mathrm{CO}$ outflows and optically seen $\mathrm{HH}$ objects (see e.g., Bachiller 1996; Hartmann 1998, and references therein). It is possible that due to this moderate nature of the shock the ionization of hydrogen could not take place. Therefore, we propose that this pair of knot-like structures is probably tracing a shock caused in the surrounding interstellar medium by 


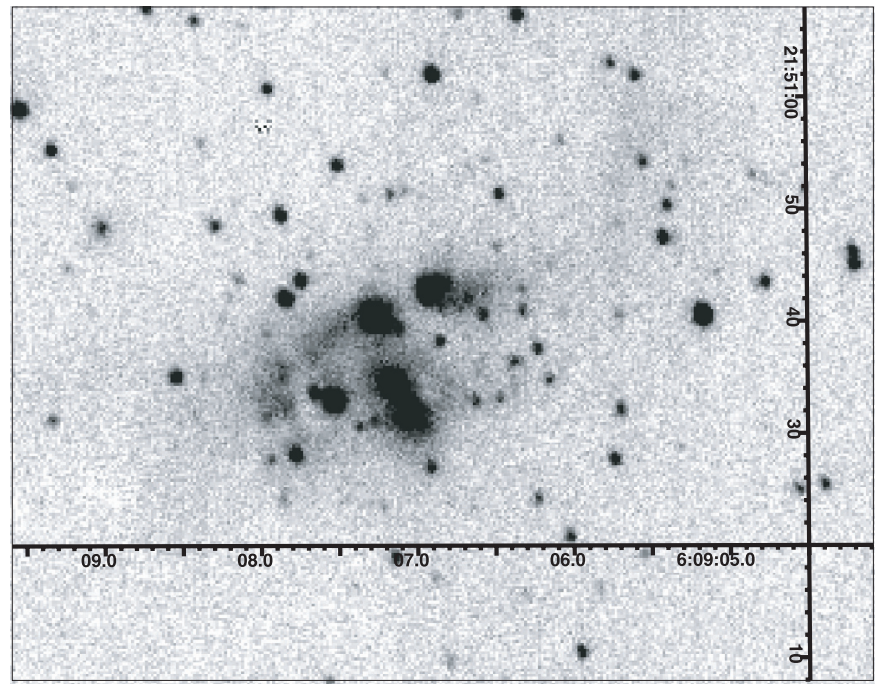

Fig. 5. $\mathrm{Br} \gamma$ line + continuum $(2.167 \mu \mathrm{m})$ image observed from TNG. North is up and East is to the left. The abscissa and ordinate are in J2000.0 epoch.

protostellar jets. We call them Knot-NW and Knot-SE respectively. These knot-like structures may be considered as infrared counterparts of $\mathrm{HH}$ Objects that are often detected in [SII] and $\mathrm{H} \alpha$ images. From the $\mathrm{H}_{2}$ image, we determine the co-ordinates $(\alpha(2000), \delta(2000))$ of the Knot-NW and Knot$\mathrm{SE}$ as $\left(6 \mathrm{~h} 9 \mathrm{~m} 5.2 \mathrm{~s},+21^{\circ} 50^{\prime} 56^{\prime \prime}\right)$ and (6h9m8.5s, $\left.+21^{\circ} 50^{\prime} 32^{\prime \prime}\right)$ respectively. Judging from the brightness of the knots, we assume that Knot-NW suffers lesser extinction and hence is tracing a blue jet and the Knot-SE the red one. Alternatively, the difference in brightness could also be due to the differences in physical parameters such as density of the two regions of interstellar medium.

There are two sources, S1 and S4, lying in the line of the jets. From the color-color diagram (Fig. 2), it appears that S1 is likely a massive member of the cluster and a Class II source, while S4 is less massive but clearly fits in the scenario of a Class I protostar. It is tempting to suggest that $\mathrm{S} 4$ is the source of the jets. It is also situated nearly in the middle of the projected distance between the two knots. The projected length of the jet is estimated to be about $0.5 \mathrm{pc}$.

\subsection{Medium resolution TNG K band spectra}

Figure 6 shows in the upper panel the normalized $K$ band spectra, taken at TNG, towards the star S1 and in the lower panel the nebular spectra obtained in a region 5 arcsec west of S1 and towards S4 (close to the path of the NW jet; see Fig. 4). Our main concern is the $\mathrm{H}_{2}$ line ratio of 2-1 S(1)/1$0 \mathrm{~S}$ (1) $(2.247 \mu \mathrm{m} / 2.122 \mu \mathrm{m})$, which depends critically on the type of excitation mechanism, namely fluorescence or shock heating (Shull \& Beckwith 1982; Draine et al. 1983; Black \& van Dishoeck 1987; Sternberg \& Dalgarno 1989; Draine \& Bertoldi 1996; Luhman et al. 1998; Walmsley et al. 2000; line ratios compiled in Glass 2000). Since the $2.247 \mu \mathrm{m}$ line is very weak and buried in the base-level noise, we put an upper limit

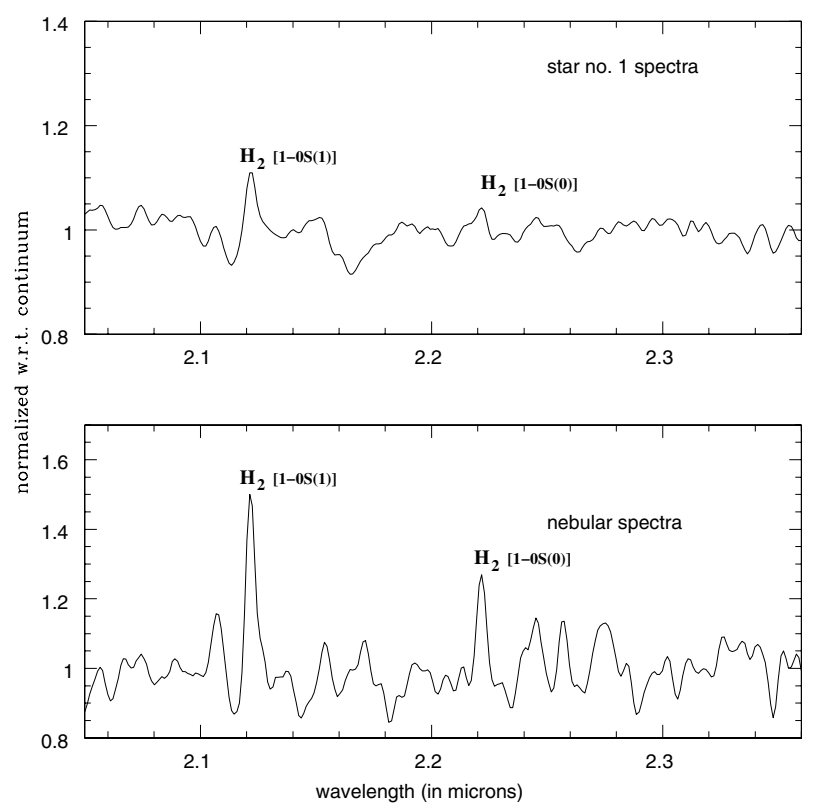

Fig. 6. Spectra towards Star 1 (top) and that of nebula (bottom) which is 5 arcsec west of Star 1 towards Star 4. Both the spectra are normalized with respect to its continuum.

for the ratio at 0.1 and 0.05 for the nebular spectra and the Star 1 respectively by taking the base line as $1.0 \pm 0.1$ for nebular spectra and $1.0 \pm 0.05$ for the Star 1 spectra. Therefore, these upper-limit ratios strongly suggest that the excitation could be due to a shock rather than fluorescence where one would expect the ratio to be $\geq 0.5$ (e.g., Black \& van Dishoeck 1987; Draine \& Bertoldi 1996). One can notice the $2.223 \mu \mathrm{m}$ line of $\mathrm{H}_{2} 1-0 \mathrm{~S}(0)$ appearing quite prominently in both the spectra with the ratio $1-0 \mathrm{~S}(0) / 1-0 \mathrm{~S}(1)$ equal to 0.55 for nebula and 0.35 for star 1 . These ratios are rather high for collisional excitation, but at an equilibrium temperature of $1000 \mathrm{~K}$, the ratio of $1-0 \mathrm{~S}(0) / 1-0 \mathrm{~S}(1)$ rises (to $\sim 0.3$ ), while the ratio of $2-$ $1 \mathrm{~S}(1) / 1-0 \mathrm{~S}(1)$ falls to insignificance (see Table 2 in Black \& van Dishoeck 1987). The fluorescence excitation populates the vibrational levels $v \geq 2$ much more than the thermal excitation; this does not seem to be the case here. We therefore infer from the spectra that the lines are likely thermally excited. The ratios for S1 are in reasonably good agreement with the ratios obtained by HLR02. Prominently missing in the spectra is the $\mathrm{Br} \gamma$ emission line at $2.167 \mu \mathrm{m}$ which could be due to the fact that the line, if present, is very weak. The absence of $\mathrm{Br} \gamma$ is also reported by HLR02. So it is clear from the presented spectra that the molecular hydrogen excitation is caused by moderate shocks (with velocities less than about $30 \mathrm{~km} \mathrm{~s}^{-1}$ that cannot cause hydrogen ionization as it requires greater than $60 \mathrm{~km} \mathrm{~s}^{-1}$ ) which explains why $\mathrm{Br} \gamma$ emission line is absent or very weak.

In Fig. 6 we find the spectrum towards S1 (upper panel) to be significantly weaker than the spectrum on the nebular region towards S4 (lower panel). So if S1 is indeed a class I protostar then we expect the two spectra to be of more or less comparable strengths (since S4 is a protostar). This is further evidence that $\mathrm{S} 1$ is likely a massive and more evolved member of the cluster. 
HLR02 concluded that the radio source KCW 188.793+1.030 (R1) corresponds to the source S1 while the spectra taken by them on the radio source KCW 188.796+1.030 (R2) do not seem to have a counterpart in their $H$ band image. But our $K^{\prime}$ image taken at Mt. Abu as well as the TNG narrow band images show that the S5 source does line up with HLR02 spectra on R2. The line ratios reported by HLR02 on R2 (S5) are nearly similar to those on $\mathrm{R} 1$ (S1). The absence of Br $\gamma$ in the two spectra presented by HLR02 and the TNG spectra shows that in general the region may be pervaded by a mild shock with velocities not greater than $30 \mathrm{~km} \mathrm{~s}^{-1}$.

\section{Conclusion}

IRAS $06061+2151$ appears to be a cluster of at least 5 bright sources, four of which seem to be early B-type YSOs. From the $J-H / H-K$ s color-color diagram we found that one of them, $\mathrm{S} 1$, could be the most massive member of the cluster rather than a background source and S4 is a Class I type star. S3 seems to be a Class II source while S2 is likely to be Class I. The IRAS source is also associated with a nebulosity. As many as 3-4 Class I (including S4 and maybe S2) and 13-14 Class II/III sources (including S1 and S3) were found towards the region of IRAS $06061+2151$ from the color-color diagram which confirms the earlier studies that the region contains large number of YSOs. A new source, S5, appears to have a very steep infrared energy distribution and is likely a massive protostar as its colors (lower limits) suggest a heavily embedded source.

From the narrow band images in $\mathrm{H}_{2}$ and $\mathrm{Br} \gamma$, two oppositely directed knot-like structures (Knot-NW and Knot-SE) have been detected. The knots are likely generated by jets from the protostellar source S4 and are the infrared counterparts of the classical $\mathrm{HH}$ objects in the optical region. From the ratios of $\mathrm{H}_{2}$ lines, we conclude that the region is probably pervaded by a mild shock or a photodissociation region that can excite these lines but is not strong enough to excite the HI lines.

Acknowledgements. We thank the referee Dr. M. Hogerheijde for valuable suggestions for improving the original version. It is a pleasure to thank the staff at Mt. Abu IR Telescope and the TNG, La Palma facility for excellent support during observations. This work is based in part on the observations made with the Italian Telescopio Nazionale Galileo at La Palma operated by the Centro Galileo Galilei of CNAA at the Observatorio del Roque de los Muchachos of the IAC, Canary Islands, Spain. D.K.O. acknowledges the support of JSPS, Japan through a fellowship. This publication makes use of data products from the 2MASS, which is a joint project of the University of Massachusetts and the Infrared Processing and Analysis
Center, funded by the NASA and the NSF. This research has made use of the SIMBAD database, operated at CDS, Strasbourg, France.

\section{References}

Anandarao, B. G. 1998, In High Energy Astrophysics (Hyderabad: Univ. Press)

Bachiller, R. 1996, ARA\&A, 34, 111

Baffa, C., Comoretto, G., Gennari, S., et al. 2001, A\&A, 378, 722

Bessel, M., \& Brett, J. M. 1988, PASP, 100, 1134

Bica, E., Dutra, C. M., \& Barbuy, B. 2003, A\&A, 397, 177

Black, J. H., \& van Dishoeck, E. F. 1987, ApJ, 322, 412

Carpenter, J. M., Snell, R. L., Schloerb, F. P., et al. 1993, ApJ, 407, 657

Carpenter, J. M., Snell, R. L., \& Schloerb, F. P. 1995a, ApJ, 445, 246

Carpenter, J. M., Snell, R. L., \& Schloerb, F. P. 1995b, ApJ, 450, 201

Chakraborty, A., Ojha, D. K., Anandarao, B. G., \& Renagarajan, T. N. 2000, A\&A, 364, 683

Davis, C. J., Eisloffel, J., \& Ray, T. P. 1994, ApJ, 426, L93

Draine, B. T., \& Bertoldi, F. 1996, ApJ, 468, 269

Draine, B. T., Roberge, W. G., \& Dalgarno, A. 1983, ApJ, 264, 485

Eisloffel, J., Smith, M. D., \& Davis, C. J. 2000, A\&A, 359, 1147

Ghosh, S. K., Iyengar, K. V. K., Karnik, A. D., et al. 2000, BASI, 28, 515

Glass, I. S. 1999, Handbook of Infrared Astronomy (Cambridge: Cambridge Univ. Press)

Gomez, M., Kenyon, S. J., \& Hartmann, L. 1994, AJ, 105, 1850

Hanson, M. M., Luhman, K. L., \& Rieke, G. H. 2002, ApJSS, 138, 35

Hartmann, L. 1998, Accretion Processes in Star Formation (Cambridge: Cambridge Univ. Press)

Herbst, T. M., Beckwith, S., \& Robberto, M. 1997, ApJ, 486, L59

Hunter, T. R., Testi, L., Taylor, G. B., et al. 1995, A\&A, 302, 249

Kenyon, S. J., Whitney, B. A., Gomez, M., et al. 1993, ApJ, 414, 773

Koornneef, J. 1983, A\&A, 128, 84

Kurtz, S. E., Churchwell, E., \& Wood, D. O. S. 1994, ApJSS, 91, 659

Lada, C. J., \& Adams, F. C. 1992, ApJ, 393, 278L

Lada, C. J., Young, E. T., \& Greene, T. P. 1993, ApJ, 408, L471

Luhman, K. L., Engelbracht, C. W., \& Luhman, M. L. 1998, ApJ, 499, 799

Meyer, M. R., Calvet, N., \& Hillenbrand, L. A. 1997, AJ, 114, 288

Nandakumar, M. S. 1999, Ph.D. Thesis, Gujarat University, Ahmedabad

Palla, F., \& Stahler, S. W. 1999, ApJ, 525, 772

Rieke, G. H., \& Labofsky, M. J. 1985, ApJ, 288, 618

Schwartz, R. D., Williams, P. M., Cohen, M., \& Jennings, D. G. 1988, ApJ, 334, 99

Shepherd, D. S., \& Churchwell, E. 1996, ApJ, 457, 267

Shull, J. M., \& Beckwith, S. 1982, ARA\&A, 20, 163

Sternberg, A., \& Dalgarno, A. 1989, ApJ, 338, 197

Stetson, P. B. 1987, PASP, 99, 191

Strom, K. M., Margulis, M., \& Strom, S. E. 1989, ApJ, 346, L33

Walmsley, C. M., Natta, A., Oliva, E., \& Testi, L. 2000, A\&A, 364, 301 